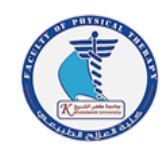

\title{
Effect of Walking on Physiological Stress in Premenstrual Syndrome: A Randomized Controlled Trial
}

\author{
Monica H. Ibrahim ${ }^{1 *}$, Amel M. Yousef ${ }^{1}$, Mohamed F. Abo Elainin ${ }^{2}$, Doaa A. Osman ${ }^{1}$ \\ ${ }^{1}$ Department of Physical Therapy for Woman's Health, Faculty of physical therapy, Cairo University, \\ Egypt. \\ ${ }^{2}$ Consultant and Chairman of Obstetrics and Gynecology, Om El-Masryeen Hospital, Egypt.
}

*Correspondence to
Monica H. Ibrahim
Department of Physical
Therapy for Woman's
Health, Faculty of Physical
Therapy, Cairo University,
Egypt.
Tel: +01285272022
Email:
monika.hany55@yahoo.com

Published online:

Sept 2020

\begin{abstract}
:
Purpose: To investigate the effect of walking on physiological stress in premenstrual syndrome (PMS).

Methods: Thirty females with PMS were randomly distributed into two equal groups. Group (A) $(n=15)$ received breathing exercises, while group (B) $(n=15)$ received the same breathing exercises in addition to walking on a treadmill. The primary outcomes were serum cortisol levels, resting heart rate (HR) and resting respiratory rate $(\mathrm{RR})$ to measure the level of physiological stress, while the secondary outcomes were daily record of severity of problems (DRSP) and abdominal pain assessed by visual analogue scale (VAS). The outcome measures were evaluated pre-treatment and after 8 weeks of treatment.

Results: Comparing both groups post-treatment revealed non-significant differences in serum cortisol levels, resting HR and DRSP $(\mathrm{P}>0.05)$, while there were significant reductions in both resting $\mathrm{RR}$ and abdominal pain VAS $(\mathrm{P}<0.05)$ in favor of group (B). The percentages of improvement post-treatment in serum cortisol levels, resting $\mathrm{HR}$, resting RR, DRSP and abdominal pain VAS were $16.05 \%, 2.92 \%, 5.19 \%, 29.73 \%$ and $25.84 \%$, respectively in group (A), while they were $25.09 \%, 7.05 \%, 15.69 \%, 31.43 \%$ and $55.30 \%$, respectively in group (B).

Conclusion: Walking is effective in treating premenstrual syndrome through reducing resting $\mathrm{RR}$ and abdominal pain intensity, as well as producing greater improvement in serum cortisol levels, resting HR and severity of PMS symptoms. Key words: Premenstrual syndrome, walking, breathing exercises, physiological stress.
\end{abstract}

\section{Introduction}

Premenstrual syndrome (PMS) describes a group of repetitive symptoms that begins at the late luteal phase of the menstrual cycle (5-7 days before menstruation) and ends in the follicular phase (2-4 days after menstruation) (1). It affects $20-80 \%$ of females of reproductive age (2).

The syndrome has severe physical, cognitive, psychological and somatic symptoms that influence activities of daily living, inter-personal relations, social life, work efficiency and educational productivity (3).

The precise pathology of PMS is unknown. It may be related to several physiological, psychological and social factors, including hormones produced by the ovaries, abnormal physiology of neurotransmitters, lifestyle habits and stress (4). Circulating cortisol is a glucocorticoid secreted from the hypothalamicpituitary-adrenal axis (HPA). Together with catecholamines, cortisol represents a valuable defense hormone and a classical stress parameter because of its response to acute physical and psychological stress (5).

Since exact PMS pathophysiology is unknown, there is no definite treatment for it, and most symptoms are being treated. In general, treatment can be divided into surgical treatment, pharmacological therapy (e.g. hormones, antidepressants and analgesics) and nonpharmacological therapy (e.g. lifestyle modifications and exercise) (6). As both surgical and pharmacological therapies have side effects, nonpharmacological therapy especially physical exercise 
has attracted the attention of health care professionals and PMS females (7).

Aerobic exercise is a physical activity of varying intensities that depends mainly on aerobic energy generating process. It improves blood flow helping cramps to go away, relaxes abdominal muscles, reduces pelvic pain and relieves pressure on nerve centers, pelvic organs and the alimentary canal (8). Additionally, it affects brain endorphins mechanisms and improves mood symptoms. Physical exercise improves PMS through elevating endorphin levels, decreasing adrenal cortisol symptoms, leading to greater pain tolerance and lesser anxiety and depression (9).

Previous studies investigated the effectiveness of aerobic exercise in reducing pain, as well as mental and physical symptoms of PMS (10-12). However, they used subjective assessment methods. Therefore, this study used objective methods to evaluate the effect of walking on physiological stress (serum cortisol levels, resting HR and resting RR) in PMS females.

\section{Patients and Methods}

\subsection{Study Design}

The study was designed as a prospective, randomized, controlled trial. Ethical approval was obtained from the institutional review board at Faculty of Physical Therapy, Cairo University. The study followed the Guidelines of Declaration of Helsinki on the conduct of human research. It was conducted between October 2019 and February 2020.

\subsection{Participants}

A sample of thirty females was recruited from the Gynecological Outpatient Clinic, Om El-Masryeen Hospital, Egypt. To be included in the study, participants were chosen sedentary, virginal, adult females suffering from PMS, which was diagnosed by a gynecologist. They had regular menstrual cycles (cycles of 21-35 days with a bleeding time of 3-10 days) and should have the following criteria in DRSP in at least one of two consecutive menstrual cycles prior to treatment starting to confirm PMS diagnosis: 1) more than three items had an average score over 3 during the luteal phase, and 2) a luteal phase score was $30 \%$ more than a follicular phase score (13). All females were non-smokers and had no traumatic life events in the last 2 months before starting the study. Their age was $18-25$ years and their body mass index (BMI) was less than $30 \mathrm{~kg} / \mathrm{m}^{2}$. Participants were excluded if they had menstrual problems (e.g. menorrhagia, metrorhagia and polycystic ovary disease), cardiorespiratory, renal, neurological and pelvic inflammatory diseases, tumors, infections, anemia, diabetes, hypertension, asthma, rheumatoid arthritis, headache, migraine, thyroid or mental disorders, participation at any other exercise training program during this study, or receiving any kind of medications, hormonal treatment or supplementation (vitamin, mineral or herbal supplement).

\subsection{Randomization}

Each female was informed about the nature, purpose and benefits of the study, the right of refusal or withdrawal at whatever time, and the confidentiality of any obtained data. Females were randomly assigned into 2 equal groups (A and B) with the use of a computer based randomization program. No dropping out of subjects from the study was reported after randomization.

\subsection{Interventions}

Group (A) included 15 females suffering from PMS who received breathing exercises for 8 weeks, while group (B) included 15 females suffering from PMS who received the same breathing exercises in addition to walking on a treadmill for 8 weeks.

\subsubsection{Breathing exercises}

All females in both groups (A \& B) received breathing exercises, 3 days per week for 8 weeks. Each female started with 5 cycles of normal breathing, then 10 cycles of deep breathing exercises, then 10 cycles of calming breathing exercises, and finally she went back to normal breathing for 10 cycles. For performing normal breathing, participants were asked to sit on a comfortable chair, maintaining good posture, with relaxed body and closed eyes. Then, they were instructed to inspire through the nose, filling first the lower part of the lungs then the middle part, then the upper part, and hold breathing for few seconds, then expire slowly with keeping relaxed abdomen and chest. These procedures were repeated for 5 cycles. For performing deep breathing exercises, participants were instructed to place one hand on the chest and one hand on the abdomen. Then, they were instructed to inspire slowly and deeply through the nose into the abdomen, feeling the rising of abdomen with this inspiration. Next, they were instructed to expire through the mouth, keeping the mouth, tongue and jaw relaxed. These procedures were repeated for 10 cycles. For performing calming breath exercises, participants were instructed to inspire slowly from the nose for 5 seconds, hold the breath for 5 seconds, and then expire slowly through the mouth or nose for 5 seconds. These procedures were repeated for 10 cycles. Throughout the exercise, breathing should be kept regular and smooth, without gulping in breaths or breathing out suddenly $(14,15)$. 


\subsubsection{Walking program on a treadmill}

All females in group (B) were engaged in a walking program on a treadmill (AC $150 \mathrm{KG} \mathrm{AX}$, Taiwan), for 30 minutes per session, 3 sessions per week, for 8 weeks. The program was started with warm up period in which each participant walked at $80 \mathrm{~m} / \mathrm{min}$ at $0.0 \%$ grade for 5 minutes. After the walk, treadmill's speed was increased to $147 \mathrm{~m} / \mathrm{min}$ and grade was increased gradually until reached $25 \%$ grade for 20 minutes. This was followed by cooling down period of 5 minutes in which the treadmill's speed and grade were decreased to $2.0 \mathrm{miles} / \mathrm{h}$ and $0.0 \%$ grade (16).

\subsection{Outcome measures}

2.5.1. Serum cortisol levels (primary outcome measure):

A blood sample was taken from each female in both groups (A \& B) before and after the treatment to measure the serum cortisol levels, which could reflect the pain intensity as decreased cortisol levels indicated improved PMS symptoms, increased pain tolerance, and decreased physiological stress (17). The cortisol levels were assessed at the late luteal phase of the menstrual cycle preceding the treatment program ( 2 days before the expected menstruation) and at the same time of the menstrual cycle after 8 weeks of the study course to evaluate the treatment effect. All blood samples were taken following overnight fasting and rest, at 8-10 a.m. to avoid diurnal variation in serum cortisol levels. Samples were collected in prechilled test tubes containing EDTA and stored at $-70^{\circ} \mathrm{C}$ until analysis. Analysis of serum cortisol levels were performed by radioinimunoassays, using Sigma-Aldich kits. The reference range of morning cortisol (from 8 am to noon) for normal adults is $6.2-19.4 \mu \mathrm{g} / \mathrm{dl}$.

\subsubsection{Resting heart rate \& resting respiratory rate (primary outcome measures):}

The HR and RR were recorded at rest, to reflect the level of physiological stress, for each female in both groups (A \& B) before and after 8 weeks of treatment through blood volume pulse sensor (BVP) which was mounted on the tip of the middle finger of the dominant hand and a respiration sensor which was placed on the female's abdomen. The participant assumed a comfortable relaxed half lying position in a quiet room; there were no tactile or auditory stimuli; there were no direct lights; there should be no restrictive clothes; soft pillows and small cushions were used to support and accommodate her body curves. Before recording any data, the subject was asked to relax through keeping her eyes and mouth gently closed and displaying any disturbing thoughts from her mind by observing and listening to her own regular breathing (in, out and a pause in between them) in order to achieve mental relaxation. After feeling relax, the resting HR and RR were recorded. The resting HR was calculated as the number of times the heart beats per minute at rest, while the resting RR was calculated as the number of breaths per minute at rest (18).

\subsubsection{Daily record of severity of problems (DRSP) (secondary outcome measure):}

It was used to confirm the diagnosis of PMS before entering in the study and to evaluate the severity of PMS symptoms before and after treatment for all females in both groups (A \& B). The DRSP included 21 separate items grouped into 11 domains in addition to 3 items describing functional impairments resulted from the symptoms. Each item was rated on the 6-point severity scales by the participant throughout her menstrual cycle. The severity levels on the DRSP were 1 (not at all), 2 (minimal), 3 (mild), 4 (moderate), 5 (severe) and 6 (extreme). The researchers gave all participants full instructions about the items of DRSP and asked them to fill it at two consecutive menstrual cycles before starting the study to confirm the diagnosis of PMS. The averages of the last 5 days before menstruation for the 11 distinct symptom items were summed to calculate the luteal phase score, and the averages of days 6-10 after menstruation onset for the 11 distinct symptom items were also summed to calculate the follicular phase score during 2 consecutive menstrual cycles before the treatment. In the beginning of the study, all participants had an average score of greater than 3 (mild) in at least three items during the luteal phase, and their luteal phase scores were 30\% higher than the follicular phase scores (13). After 8 weeks of the study course, the participants filled the DRSP and the luteal phase score was calculated to evaluate the treatment effect.

\subsubsection{Abdominal pain assessed by VAS (secondary outcome measure):}

The abdominal pain intensity was assessed for each female in both groups (A \& B) before and after treatment through the VAS. The pain VAS is onedimensional measure of pain intensity, which has been commonly used in various adult populations. It is a $10-\mathrm{cm}$ horizontal line on which the participant's pain intensity was represented by a point between the extremes of "no pain at all" and "worst imaginable pain". Each participant was asked to mark a point on the VAS line between the extremes that related to her current abdominal pain intensity. Then, the centimeters were measured in each time from the left end of the line to the marked point to obtain the VAS score for abdominal pain intensity (19). 
Prior for final analysis, data were screened, for normality assumption test by using Shapiro-Wilk test $(\mathrm{P}>0.05)$ and homogeneity of variance by Levene's test $(\mathrm{P}>0.05)$. The data was normally distributed and parametric analysis is done. The statistical analysis was conducted by using statistical SPSS Package program version 25 for Windows (SPSS, Inc., Chicago, IL). All data were expressed as mean and standard deviation for all outcomes variables. Paired t-test to compare between pre-and post-treatment within each group and using unpaired (independent) t-test to compare between two groups. All statistical analyses were significant at level of probability less than an equal $0.05(\mathrm{P} \leq 0.05)$.

\section{Results}

Both groups were similar at baseline $(\mathrm{P}>0.05)$ regarding age, BMI, menstrual cycle length, number of menstrual cycles in the last year and all outcome measures (Tables 1-2).

The serum cortisol levels revealed a statistically significant reduction within both groups (A \& B) $(\mathrm{P}<0.05)$. The post-treatment comparison of both groups showed a statistically non-significant difference $(\mathrm{P}>0.05)$. However, there was a greater percentage of improvement with regard to serum cortisol levels in group (B) (25.09\%) than in group (A) $(16.05 \%)$ (Table 2$)$.

The resting HR revealed a statistically significant reduction within both groups $(\mathrm{A} \& \mathrm{~B})(\mathrm{P}<0.05)$. The post-treatment comparison of both groups showed a statistically non-significant difference $(\mathrm{P}>0.05)$. However, there was a greater percentage of improvement with regard to resting HR in group (B) (7.05\%) than in group (A) $(2.92 \%)$ (Table 2).

The resting RR revealed a statistically nonsignificant difference within group (A) $(\mathrm{P}>0.05)$, while it revealed a statistically significant reduction within group (B) $(\mathrm{P}<0.05)$. The post-treatment comparison of both groups showed a statistically significant reduction $(\mathrm{P}<0.05)$ in favour of group $(\mathrm{B})$. Also, there was a greater percentage of improvement with regard to resting RR in group (B) (15.69\%) than in group (A) (5.19\%) (Table 2).

The DRSP scores revealed a statistically significant reduction within both groups $(\mathrm{A} \& \mathrm{~B})(\mathrm{P}<0.05)$. The post-treatment comparison of both groups showed a statistically non-significant difference $(\mathrm{P}>0.05)$. However, there was a greater percentage of improvement about DRSP scores in group (B) $(31.43 \%)$ than in group (A) $(29.73 \%)$ (Table 2$)$.

The abdominal pain VAS revealed a statistically significant reduction within both groups (A \& B) $(\mathrm{P}<0.05)$. The post-treatment comparison of both groups showed a statistically significant reduction $(\mathrm{P}<0.05)$ in favour of group $(\mathrm{B})$. Also, there was a greater percentage of improvement with regard to abdominal pain VAS in group (B) $(55.30 \%)$ than in group (A) (25.84\%) (Table 2).

Table (1): Baseline characteristics of females in both groups

\begin{tabular}{|c|c|c|c|}
\hline & Group (A) & Group (B) & \multirow{2}{*}{$P$ value } \\
\hline & $\overline{\mathbf{x}} \pm \mathbf{S D}$ & $\overline{\mathbf{x}} \pm \mathrm{SD}$ & \\
\hline Age (years) & $20.93 \pm 2.28$ & $20.67 \pm 2.38$ & $0.756^{\mathrm{Ns}}$ \\
\hline BMI $\left(\mathrm{Kg} / \mathrm{m}^{2}\right)$ & $23.87 \pm 2.49$ & $24.48 \pm 2.77$ & $0.530^{\mathrm{NS}}$ \\
\hline $\begin{array}{l}\text { Menstrual cycle } \\
\text { length (Days) }\end{array}$ & $25.40 \pm 1.76$ & $26.47 \pm 1.68$ & $0.102^{\mathrm{NS}}$ \\
\hline $\begin{array}{l}\text { Number of menstrual } \\
\text { cycles in the last year }\end{array}$ & $14.00 \pm 0.92$ & $13.53 \pm 0.83$ & $0.158^{\mathrm{NS}}$ \\
\hline
\end{tabular}

Table 2. The serum cortisol levels, resting HR, resting RR, DRSP and abdominal pain VAS for both groups

\begin{tabular}{|c|c|c|c|c|}
\hline & Group (A) & Group (B) & \multirow{2}{*}{ P value* } \\
\hline & & $\overline{\mathbf{x}} \pm$ SD & $\overline{\mathbf{x}} \pm$ SD & \\
\hline \multirow{4}{*}{$\begin{array}{l}\text { Serum cortisol } \\
(\mu \mathrm{g} / \mathrm{dl})\end{array}$} & Pre-treatment & $19.07 \pm 3.32$ & $19.37 \pm 4.44$ & $0.839^{\mathrm{NS}}$ \\
\hline & Post-treatment & $16.01 \pm 3.34$ & $14.51 \pm 4.41$ & $0.306^{\mathrm{NS}}$ \\
\hline & Improvement $\%$ & $16.05 \%$ & $25.09 \%$ & \\
\hline & $\mathrm{P}$ value** & $0.001^{\mathrm{s}}$ & $0.0001^{\mathrm{s}}$ & \\
\hline \multirow{4}{*}{$\begin{array}{l}\text { Resting HR } \\
\text { (beats/min) }\end{array}$} & Pre-treatment & $75.40 \pm 7.77$ & $75.60 \pm 7.88$ & $0.945^{\mathrm{NS}}$ \\
\hline & Post-treatment & $73.20 \pm 6.13$ & $70.27 \pm 5.89$ & $0.193^{\text {NS }}$ \\
\hline & Improvement $\%$ & $2.92 \%$ & $7.05 \%$ & \\
\hline & $\mathrm{P}$ value** & $0.021^{\mathrm{S}}$ & $0.0001^{\mathrm{s}}$ & \\
\hline \multirow[t]{4}{*}{$\begin{array}{l}\text { Resting RR } \\
\text { (breaths/min) }\end{array}$} & Pre-treatment & $14.27 \pm 1.86$ & $14.47 \pm 1.30$ & $0.737^{\mathrm{NS}}$ \\
\hline & Post-treatment & $13.53 \pm 1.06$ & $12.20 \pm 0.56$ & $0.001^{\mathrm{s}}$ \\
\hline & Improvement \% & $5.19 \%$ & $15.69 \%$ & \\
\hline & $\mathrm{P}$ value** & $0.119^{\mathrm{NS}}$ & $0.0001^{\mathrm{s}}$ & \\
\hline \multirow[t]{4}{*}{$\begin{array}{l}\text { DRSP (Luteal } \\
\text { phase score) }\end{array}$} & Pre-treatment & $0.74 \pm 0.03$ & $0.70 \pm 0.06$ & $0.190^{\mathrm{NS}}$ \\
\hline & Post-treatment & $0.52 \pm 0.09$ & $0.48 \pm 0.04$ & $0.099^{\mathrm{NS}}$ \\
\hline & Improvement \% & $29.73 \%$ & $31.43 \%$ & \\
\hline & $\mathrm{P}$ value** & $0.0001^{\mathrm{s}}$ & $0.0001^{\mathrm{s}}$ & \\
\hline \multirow[t]{4}{*}{$\begin{array}{l}\text { Abdominal } \\
\text { pain VAS }\end{array}$} & Pre-treatment & $3.87 \pm 0.91$ & $3.87 \pm 0.92$ & $1.000^{\mathrm{NS}}$ \\
\hline & Post-treatment & $2.87 \pm 0.99$ & $1.73 \pm 0.70$ & $0.001^{\mathrm{s}}$ \\
\hline & Improvement \% & $25.84 \%$ & $55.30 \%$ & \\
\hline & $\mathrm{P}$ value** & $0.004^{\mathrm{s}}$ & $0.0001^{\mathrm{s}}$ & \\
\hline
\end{tabular}

\footnotetext{
Data were expressed as mean \pm standard deviation

Inter-group comparison; $* *$ intra-group comparison of the results pre- and post-treatment.

${ }_{\text {NS }} \mathrm{P}>0.05=$ non-significant, ${ }^{\mathrm{S}} \mathrm{P}<0.05=$ significant, $\mathrm{P}=$ Probability
}

\section{Discussion}

Premenstrual Syndrome (PMS) is a disorder that is frequently seen in young adult females. It has a negative influence on young females' lives, which can lead to reduced work efficiency and quality of work, increased accidents and lack of school attendance. As a result, there is a need to develop treatment protocols for coping with premenstrual 
symptoms (20). Therefore, this study aimed to examine the effect of walking on physiological stress in PMS.

Regarding serum cortisol levels, results showed a statistically significant reduction within both groups (A \& B). The post-treatment comparison of both groups showed a statistically non-significant difference. However, there was a greater percentage of improvement in group (B) than in group (A). These results indicated the positive impact of breathing exercises with or without walking program on reducing serum cortisol levels in PMS females. These findings agree with $\mathrm{Ma}$ et al. (21) who investigated the effectiveness of deep breathing exercises for 20 sessions, respectively, on reducing salivary levels of cortisol in healthy adult subjects. Additionally, Martarelli et al. (22) reported that relaxation promoted by diaphragmatic breathing resulted in decreased cortisol levels in athletes following exhaustive exercise. The lowered cortisol levels in response to breathing exercise could be related to the close association between cortisol and HPA axis, which can easily be affected by breathing (23).

The greater improvement percentage in group (B) than in group (A) reflected the beneficial effect of walking program on a treadmill on lowering serum cortisol levels in PMS females. This result can be supported with Beserra et al. (24) who revealed a positive effect of physical exercising on reducing cortisol concentrations in individuals with major depressive disorder. Moreover, sensitivity analysis and subgroup analyses showed an influence of exercise type (aerobic exercise) on lowering cortisol concentrations. Also, de Souza et al. (25) found a reduction trend in blood cortisol levels in response to daily moderate to high intensity aerobic exercise for 5 consecutive days. These findings highlight an adaptive hormonal mechanism in response to repeated stress, as shown by reduced cortisol levels. In addition, Nabkasorn et al. (26) showed reduced urinary cortisol levels following 8 weeks of physical training in depressed females aged 18-20 years. Moreover, Rimmele et al. (27) reported that trained men displayed significantly lower cortisol levels in response to stressors than untrained counterparts. Furthermore, Webb et al. (28) concluded that individuals having high aerobic fitness exhibit reduced cortisol responses to a combined challenge of physical and mental stress. The stress-relieving result of exercise could be mediated by the decrease in the subject's neuromuscular hyperactivity in the HPA axis (29).

Regarding resting HR, results showed a statistically significant reduction within both groups (A \& B). The post-treatment comparison of both groups showed a statistically non-significant difference.
However, there was a greater percentage of improvement in group (B) than in group (A). These results indicated the favorable effect of breathing exercises with or without walking program on decreasing resting HR in females having PMS. These results are consistent with studies that found a significant reduction in HR in PMS females after 3 months of breathing exercises practice in the form of pranayama $(30,31)$. Females with PMS have sympathovagal imbalance with shifting of autonomic balance in favor of sympathetic activity and these females are unable to cope up with stressful situations. Thus, practicing breathing exercises improves autonomic function by altering parasympathetic and sympathetic activities (32).

The greater improvement percentage in group (B) than in group (A) revealed the advantageous effect of aerobic walking on reducing resting HR in females with PMS. This finding is in line with a systematic review and meta-analysis which showed that increased physical activity is accompanied by decreased HR in middle aged adults (33). Also, Nabkasorn et al. (26) showed reduced HR after 8 weeks of physical training in females having depressive symptoms. Additionally, Rimmele et al. (27) reported that trained men exhibited significantly lower HR in response to stressors than untrained men. Moreover, de Geus et al. (34) demonstrated that aerobic fitness was connected to lower HR. The reduced HR after regular exercise or physical activity in humans could be related to a reduction in the intrinsic HR via mechanisms which have not yet been fully recognized (35).

Regarding resting RR, results showed a statistically non-significant difference within group (A), while there was a statistically significant reduction within group (B). The post-treatment comparison of both groups showed a statistically significant reduction in favor of group (B).

The non-significant difference within group (A) revealed that 8 weeks of breathing exercise was not effective in reducing resting RR in PMS females. This result could be related to the short duration of breathing exercise practice in the current study as previous studies revealed that 3 months of breathing exercises in the form of pranayama was effective in inducing RR lowering in PMS females and in normal healthy individuals $(31,36)$.

The significant decrease in resting $R R$ in favor of group (B) post-treatment revealed the effectiveness of walking program. This result is consistent with Gulam (18) who stated that resting RR is decreased after physical training. The resting RR is about $12-20$ breaths/minute in normal untrained individual, whereas it comes down to 7-8 breaths/minute in trained athletes or individuals. This reduction in 
resting RR reflects greater respiratory efficiency resulted by training or physical exercises.

Regarding DRSP scores, results revealed that there was a statistically significant reduction within both groups (A \& B). The post-treatment comparison of both groups revealed a statistically non-significant difference. However, there was a greater percentage of improvement in group (B) than in group (A). These results demonstrated breathing exercises efficacy with or without walking program on reducing PMS symptoms severity in adult females. These results can be supported with Abay and Kaplan (37) who reported that different stress coping methods, such as breathing exercises, significantly facilitates PMS management as stress intensifies PMS severity.

The greater percentage of improvement in group (B) than in group (A) revealed the valuable effect of aerobic walking program on reducing PMS severity. These results coincide with former studies reporting the improving effect of aerobic exercise on PMS symptoms total score, physical symptoms, anxiety and depressive symptoms in females having PMS $(38,39)$. Increased serum aldosterone and prostaglandin $\mathrm{E}_{2}$, as well as decreased magnesium and vitamin $\mathrm{B}_{6}$ are factors involved in some physical symptoms like headache, swelling, weight gain and breast pain (40). Physical activity can reduce serum aldosterone and provoke sodium and water reabsorption (41), resulting in physical symptoms improvement. Mood changes in PMS females may be due to the influence of estrogen and progesterone on the serotonin, $\gamma$-aminobutyric acid and dopamine systems (42). Physical activity has neuroimmunomodulatory impact, increases neurotrophies and $\beta$-endorphins levels, reduces sympathetic response, influences HPA axis reactions, enhances serotonin system, corrects body image and promotes self-efficacy, which can improve selfesteem and self-concept; all of these effects may reduce anxiety and depression (3).

Regarding abdominal pain VAS, results showed a statistically significant reduction within both groups (A \& B). The post-treatment comparison of both groups revealed a statistically significant reduction in favor of group (B). The pain-relieving effect of breathing exercises can be supported with a recent study that revealed the effectiveness of breathing exercises in reducing VAS scores in primipara. Breathing exercises enhances endorphins release and promotes relaxation, especially abdominal muscles relaxation, resulting in elimination of pain feelings (43). The significant analgesic effect of the walking program in the current study can be confirmed with previous studies that revealed prostaglandins improvement in response to aerobic exercise, helping to reduce abdominal pain in dysmenorrheic females $(44,45)$.

The main limitation of the current study was the inability to study the long-term effect of walking and reappearance of symptoms after stopping walking program.

\section{Conclusion}

Walking is beneficial in reducing the level of physiological stress (serum cortisol levels, resting heart rate and resting respiratory rate), severity of PMS symptoms and abdominal pain intensity in adult females with premenstrual syndrome.

\section{Funding}

Not funded.

\section{Conflict of Interest}

Authors declare no potential conflicts of interest.

\section{Acknowledgments}

Authors would like to thank all females who participated in the current study.

\section{References}

1. Hasani N, Kazemi M, Afshar H, Kazemi M, Tavakoli M. Comparison of the effects of relaxation and vitamin B6 on emotional and physical symptoms in premenstrual syndrome. Evid Based Care J 2015; 5(15):75-83.

2. Khajehei M. Etiology, diagnosis and management of premenstrual syndrome. J Pain Relief 2015; 4(4):193-200.

3. ElDeeb AM, Atta HK, Osman DA. Effect of whole body vibration versus resistive exercise on premenstrual symptoms in adolescents with premenstrual syndrome. Bull Fac Phys Ther 2020; 25(1).

4. Watanabe K, Shirakawa T. Characteristics of perceived stress and salivary levels of secretory immunoglobulin A and cortisol in Japanese women with premenstrual syndrome. Nurs Midwifery Stud 2015; 4(2):e24795.

5. Tanaka Y. Rapid analysis of stress response material in the saliva. J Clin Lab Med 2008; 52:441-454.

6. Abedy H, Neksereshgt A, Tashakoriyan F. The effects of resistance and endurance exercise on physical and psychobehavioral symptoms of premenstruation syndrome. Pars J Med Sci 2014; 12(3):9-14. 
7. Daley A. Exercise and premenstrual symptomatology: a comprehensive review. J Women's Health (Larchmt) 2009; 18(6):895899.

8. Ghanbari Z, Manshavi FD, Jafarabadi M. The effect of three months regular aerobic exercise on premenstrual syndrome. J Fam Reprod Health 2008; 2(4):167-171.

9. Karimian N, Rezaeian M, Nassaji F. The effects of physical activity on premenstrual syndrome. $\mathrm{J}$ Med Sci Zanjan 2006; 13(53):8-15.

10. Dehnavi Z, Rastaghi S, Rad M. A survey on the association of premenstrual syndrome with type of temperament in high school students. IJOGI 2017; 20(5):15-23.

11. Jafarnejad F, Dehnavi Z, Mojahedi M, Shakeri M, Sardar M. Effect of aerobic exercise program on premenstrual syndrome in women of hot and cold temperaments. J Babol Univ Med Sci 2016; 18(8):54-60.

12. Sabaei Y, Sabaei S, Khorshidi D, Ebrahimpour S, Fallah-Rostami F. The association between premenstrual syndrome and physical activity and aerobic power in female high school students. Crescent J Med Biol Sci 2015; 2(2):53-58.

13. Endicott J, Nee J, Harrison W. Daily Record of Severity of Problems (DRSP): reliability and validity. Arch Womens Ment Health 2006; 9(1):41-49.

14. Davis M, Eshelman ER, McKay M. Breathing: The Relaxation and Stress Reduction Workbook Chapter Singles. Oakland, CA: New Harbinger Publications, 2008.

15. Bourne EJ. The Anxiety and Phobia Workbook. $5^{\text {th }}$ Ed., Oakland, CA: New Harbinger Publications, 2010.

16. Riebe D, Ehrman JK, Liguori G, Magal M. General Principles of Exercise Prescription. In: Ehrman JK, Liguori G, Magal M, Riebe D, American College of Sports Medicine, editors. ACSM's Guidelines for Exercise Testing and Prescription. $10^{\text {th }}$ Ed., Philadelphia, PA: Wolters Klumer, 2018.

17. Omidali F. The effect of Pilates exercise and consuming fennel on premenstrual syndrome symptoms in non-athletic girls. Complement Med J 2015; 2(15):1203-1213.

18. Gulam A. Impact of exercises on muscular, respiratory and circulatory body systems. Int $\mathbf{J}$ Acad Res Dev 2016; 1(12):59-62.

19. Hawker GA, Mian S, Kendzerska T, French M. Measures of adult pain: Visual Analog Scale for Pain (VAS Pain), Numeric Rating Scale for Pain (NRS Pain), McGill Pain Questionnaire (MPQ), Short-Form McGill Pain Questionnaire (SFMPQ), Chronic Pain Grade Scale (CPGS), Short
Form-36 Bodily Pain Scale (SF-36 BPS), and Measure of Intermittent and Constant Osteoarthritis Pain (ICOAP). Arthritis Care Res (Hoboken) 2011; 63 Suppl 11:S240-252.

20. Yilmaz-Akyuz E, Aydin-Kartal Y. The effect of diet and aerobic exercise on premenstrual syndrome: randomized controlled trial. Revista de Nutrição 2019; 32.

21. Ma X, Yue ZQ, Gong ZQ, Zhang H, Duan NY, Shi YT, Wei GX, Li YF. The effect of diaphragmatic breathing on attention, negative affect and stress in healthy adults. Front Psychol 2017; 8:874.

22. Martarelli D, Cocchioni M, Scuri S, Pompei P. Diaphragmatic breathing reduces exerciseinduced oxidative stress. Evid Based Complement Alternat Med 2011; 2011:932430.

23. Argyropoulos SV, Bailey JE, Hood SD, Kendrick AH, Rich AS, Laszlo G, Nash JR, Lightman SL, Nutt DJ. Inhalation of $35 \% \mathrm{CO}(2)$ results in activation of the HPA axis in healthy volunteers. Psychoneuroendocrinology 2002; 27(6):715-729.

24. Beserra AHN, Kameda P, Deslandes AC, Schuch FB, Laks J, Moraes HS. Can physical exercise modulate cortisol level in subjects with depression? A systematic review and metaanalysis. Trends Psychiatry Psychother 2018; 40(4):360-368.

25. de Souza HS, Jardim TV, Barroso WKS, de Oliveira Vitorino PV, Souza ALL, Jardim PCV. Hormonal assessment of participants in a long distance walk. Diabetol Metab Syndr 2019; 11:19.

26. Nabkasorn C, Miyai N, Sootmongkol A, Junprasert S, Yamamoto H, Arita M, Miyashita $\mathrm{K}$. Effects of physical exercise on depression, neuroendocrine stress hormones and physiological fitness in adolescent females with depressive symptoms. Eur J Public Health 2006; 16(2):179-184.

27. Rimmele U, Zellweger BC, Marti B, Seiler R, Mohiyeddini C, Ehlert U, Heinrichs M. Trained men show lower cortisol, heart rate and psychological responses to psychosocial stress compared with untrained men. Psychoneuroendocrinology 2007; 32(6):627635.

28. Webb HE, Rosalky DS, Tangsilsat SE, McLeod KA, Acevedo EO, Wax B. Aerobic fitness affects cortisol responses to concurrent challenges. Med Sci Sports Exerc 2013; 45(2):379-386.

29. Silverman MN, Deuster PA. Biological mechanisms underlying the role of physical fitness in health and resilience. Interface Focus 2014; 4(5):20140040. 
30. Goyal R, Goyal PR, Garg R. Effect of pranayama on blood pressure and heart rate in premenstrual syndrome. J Evolution Med Dent Sci 2016; 5(73):5335-5337.

31. Sharma B, Misra R, Singh K, Sharma R, Archana. Comparative study of effect of anuloma-viloma (pranayam) and yogic asanas in premenstrual syndrome. Indian $\mathbf{J}$ Physiol Pharmacol 2013; 57(4):384-389.

32. Kulshreshtha M, Kumar Y, Agarwal V, Dhama V. Symathovagal imbalance in premenstrual syndrome. Indian J Physiol Pharmacol 2013; 57(4):443-447.

33. Reimers AK, Knapp G, Reimers CD. Effects of Exercise on the Resting Heart Rate: A Systematic Review and Meta-Analysis of Interventional Studies. J Clin Med 2018; 7(12):503.

34. de Geus EJ, van Doornen LJ, Orlebeke JF. Regular exercise and aerobic fitness in relation to psychological make-up and physiological stress reactivity. Psychosom Med 1993; 55(4):347-363.

35. Bahrainy S, Levy WC, Busey JM, Caldwell JH, Stratton JR. Exercise training bradycardia is largely explained by reduced intrinsic heart rate. Int J Cardiol 2016; 222:213-216.

36. Sunitha G, Ravi BN. Effect of deep breathing on respiratory parameters in healthy young individuals. J Evol Med Dent Sci 2013; 2(19):3305-3312.

37. Abay H, Kaplan S. Current approaches in premenstrual syndrome management. Bezmialem Science 2019; 7(2):150-156.

38. Samadi Z, Taghian F, Valiani M. The effects of 8 weeks of regular aerobic exercise on the symptoms of premenstrual syndrome in nonathlete girls. Iran J Nurs Midwifery Res 2013; 18(1):14-19.

39. Dehnavi ZM, Jafarnejad F, Goghary SS. The effect of 8 weeks aerobic exercise on severity of physical symptoms of premenstrual syndrome: a clinical trial study. BMC Womens Health 2018; 18(1):80.

40. Rapkin AJ, Akopians AL. Pathophysiology of premenstrual syndrome and premenstrual dysphoric disorder. Menopause Int 2012; $18(2): 52-59$.

41. Wilmore JH, Costill DL. Physiology of sport and exercise. Translated by Moeini. Tehran: Mobtakeran Publishers, 2008.

42. Del Río JP, Alliende MI, Molina N, Serrano FG, Molina S, Vigil P. Steroid hormones and their action in women's brains: the importance of hormonal balance. Front Public Health 2018; $6: 141$.
43. Marzouk T, Emarah HA. Effectiveness of Breathing Exercise on Reducing Pain Perception and State Anxiety among Primi Parturients. IOSR Journal of Nursing and Health Science 2019; 8(2):15-22.

44. Matthewman G, Lee A, Kaur JG, Daley AJ. Physical activity for primary dysmenorrhea: a systematic review and meta-analysis of randomized controlled trials. Am J Obstet Gynecol 2018; 219(3):255.e1-255.e20.

45. Abbaspour Z, Rostami M, Najjar S. The effect of exercise on primary dysmenorrhea. J Res Health Sci 2006; 6(1):26-31. 